\title{
Investigations on Harmonics in Smart Distribution Grid with Solar PV Integration
}

\author{
J. V. Desai ${ }^{1}$. Pradeep Kumar Dadhich ${ }^{2}$ P. K. Bhatt ${ }^{1}$
}

Received: 28 January 2016 / Accepted: 25 July 2016 / Published online: 8 August 2016

C) Springer Science+Business Media Singapore 2016

\begin{abstract}
Integration of solar PV into the grid poses many challenges in the power system. One such serious challenge is harmonics penetration into the system. Harmonics are generated by the solar PV and the associated power electronics circuitry. Moreover, the loads connected to the distribution system are usually nonlinear and harmonic rich in nature. Therefore, a lot of harmonics gets introduced into the system from the source as well from load ends, and thereby affecting the power system performance. In this paper, harmonic distortion issues associated with solar $\mathrm{PV}$ integration are analyzed considering the limits of IEEE 519-1992 standard. The Newton-Raphson algorithm is modified with solar PV integration for harmonic power flow study. The formulated approach is employed to determine the discrete harmonic components, total harmonic distortion (THD) levels, and values of the system power flow variables. The developed approach is tested on a smart radial distribution feeder and the results show that with solar PV integration, the harmonic distortion increases above acceptable limits. In order to have a vision of smart grids for providing clean power supply, the authors feel that there is a serious need to study the issues related to harmonic generation, and further to develop control strategies to eliminate them.
\end{abstract}

\section{P. K. Bhatt}

pramod_bhatt19@rediffmail.com

1 College of Engineering \& Technology, Mody University, Lakshmangarh, India

2 Integrated Research and Action for Development, New Delhi, India
Keywords Harmonics - Total harmonic distortion . Harmonic power flow · Grid integrated solar PV · Smart grid distribution system

\section{Introduction}

The presence of harmonics is unavoidable in a power system due to the nonlinear characteristics of equipment and connected loads [1]. Integration of solar PV system further intensifies the harmonics in the distribution network. These harmonics emanate within the inverter during the normal power conversion process [2]. The nonlinear behavior of solar PV with changing weather condition increases inverter switching, which injects additional harmonics in the system $[3,4]$. The magnitude and order of harmonics depend on the power inverter technology used, adopted modulation technique, method of commutation, existence of high or low frequency coupling transformer, interconnection configuration and filters used at the output side of the inverter [5-9].

The interaction between grid components and number of solar PV units further amplifies the harmonic distortion [10].

The objective of this paper is to analyze the distribution network with solar PV integration, and to study the voltage/current harmonic distortions at various buses/branches. Paper also deals with the deviation of these distortions from the standard IEEE limits [11], thereby emphasizes the need for developing suitable control strategies to eliminate them in order to maintain the clean power supply. To examine these issues, the Newton-Raphson algorithm is reformulated and implemented by including the solar PV and nonlinear loads on the smart radial distribution feeder. The results 
Fig. 1 a Equivalent circuit, Homo junction solar cell. $\mathbf{b}$ Equivalent circuit, Solar cell with impurity recombination current

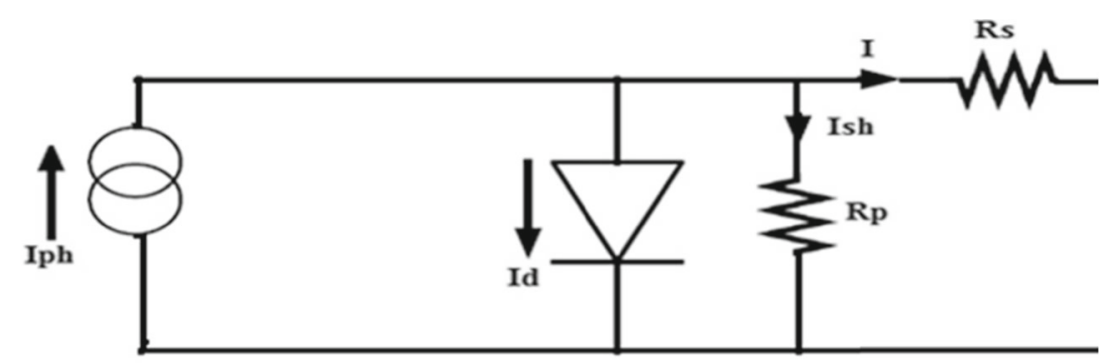

(a) Equivalent circuit, Homo junction solar cell

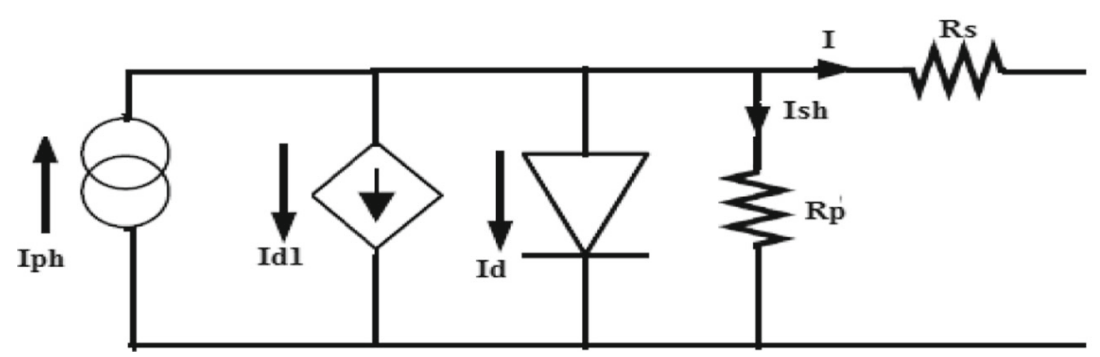

(b) Equivalent circuit, Solar cell with impurity recombination current

of the harmonic power flow analysis are comprehensively analyzed and the observations are presented.

The paper is organized as follows: The modeling of various power system components in harmonic domain is presented in "Modeling of Power System Components". The harmonic analysis and simulation algorithm is discussed in "Harmonic Analysis Algorithm". Design of smart radial distribution grid is given in "Design of Smart Distribution Grid System". "Simulation Results Analysis" presents simulation results analysis and associated discussion on the harmonic distortions. Finally, "Conclusion" concludes the paper by summarizing the work carried and investigations made.

\section{Modeling of Power System Components}

For analyzing the issues connected with the harmonics, we need to develop appropriate models of various power system components, used in our study. This section presents the harmonic domain modeling of various power system components.

\section{Modeling of Solar PV}

A PV system exhibits the nonlinear I-V characteristics and introduces harmonics in the system by itself. An ideal PV cell is modeled as a current source which represents the generated current due to incident light and a parallel diode for p-n junction of PV cell but the model of a real PV cell contains additional parallel resistance $\mathrm{R}_{\mathrm{p}}$, represents the current leakage through the cell and a series resistance $R_{s}$, accounts for an extra voltage drop between the junction as shown in Fig. 1a. In an ideal situation, to determine the upper limit of open circuit voltage and the efficiency of solar cell, it is assumed that only radiative recombination takes place. But In the actual solar cell, the recombination via impurities predominates as presented in Fig. 1b [12-15]. Furthermore, the presence of solar array capacitance, as presented in Fig. 2, also increases the voltage ripple and has the dominant effect
Fig. 2 Equivalent circuit with solar array capacitance

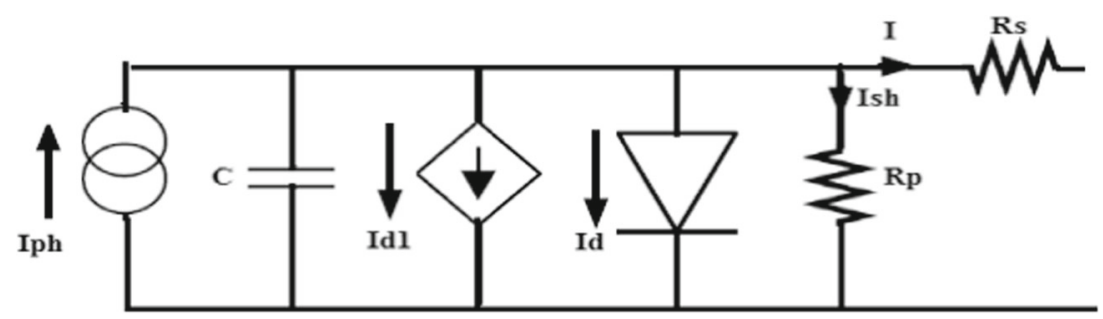


Fig. 3 Transformer equivalent circuit for harmonics studies

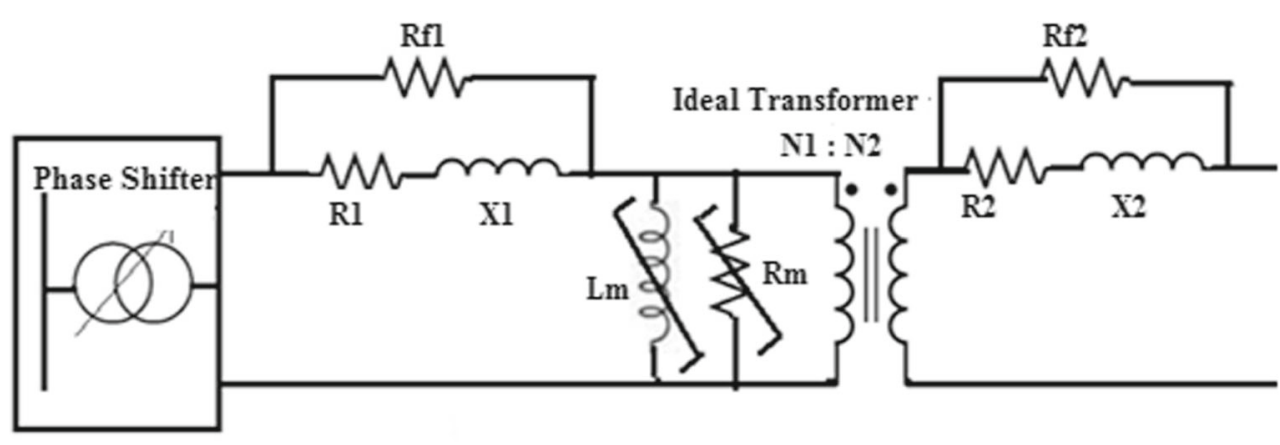

on the performance of the switching regulators at higher switching frequencies $[16,17]$.

The equation for current and voltage relationship of PV array is expressed in Eq. 1.

$I=I_{p h}-I_{0}\left[\exp \left(\frac{q\left(V+R_{s} I\right)}{N_{s} K T}\right)-1\right]-\left(\frac{V+R_{s} I}{R_{p}}\right)$

Where, $I_{p h}$ : Photo generated current (A) i.e. current generated by the incident light (it is directly proportional to the sun irradiation), $I_{d}$ : Diode equation (A), $I_{0}:$ Reverse saturation or leakage current of the diode (A), $q$ : Charge of electron $\left(1.160217646 \times 10^{-19} \mathrm{C}\right), K$ : Boltzmann constant $\left(1.3806503 \times 10^{-23} \mathrm{~J} / \mathrm{K}\right), T$ : Temperature of the $\mathrm{p}-\mathrm{n}$ junction ( ${ }^{\circ}$ Kelvin), $N_{s}$ : Number of cells, $R_{s}$ : Series resistance $(\Omega), R_{p}$ : Parallel resistance $(\Omega), I_{d 1}$ : Recombination current.

\section{Modeling of Transformer}

The shunt branch is the main source of harmonics in transformer. These harmonics are produced due to nonlinear core loss resistance, nonlinear inductance and frequency dependent resistances of primary and secondary windings. Fig. 3 represents the transformer equivalent circuit containing phase shifter and electrical equivalents of harmonic producing elements. Moreover, the generated harmonics also depends on the phase shifting and transformer winding connections $[18,19]$.

Where,

R1, R2 Primary and secondary winding resistance representing copper losses,

$\mathrm{X} 1, \mathrm{X} 2$ Primary and secondary winding reactance representing leakage flux,

Lm Magnetizing reactance, representing core flux

$\mathrm{Rm} \quad$ Magnetizing resistance representing Iron loss in the core

Rf1, Rf2 Primary and secondary winding resistances depending on frequency, represents skin effect

N1: N2 Ideal transformer turns ratio
The equivalent $\pi$ harmonic model of transformer can be developed as shown in Fig. 4 [20-22].

Where, $Y_{A}^{(h)}, Y_{B}^{(h)}, Y_{C}^{(h)}$ are equivalent admittance of transformers after considering all the elements and harmonic producing branches. The harmonics admittance matrix for complete transformer is expressed in Eq. 2.

$\left[Y_{x y(t r)}^{(h)}\right]=\left[\begin{array}{cc}\left(Y_{A}^{(h)}+Y_{B}^{(h)}\right) & -Y_{A}^{(h)} \\ -Y_{A}^{(h)} & \left(Y_{C}^{(h)}+Y_{A}^{(h)}\right)\end{array}\right]$

\section{Modeling of Distribution Lines and Cables}

Mathematical modeling of power systems in frequency and time domain techniques employ an admittance matrix model of the system. For balanced system the admittance matrix is defined as $[24,25]$.

$\left[Y_{\text {bus }}\right]=[A] \cdot\left[Y_{\text {prim }}\right][A]^{T}$

In Eq. 3, Incidence matrix $[A]$ is used to represent network connectivity.

An un-transposed, LV distribution feeder feeds the loads which are highly nonlinear and contains harmonics. In order to analyze these conditions, a harmonic model of the power system network is required [23]. For non-sinusoidal operating conditions the admittance matrix will be defined at harmonic frequencies. One such LV three phase, four wire system is shown in Fig. 5, in which the conductors are connected between the buses $x$ and $y$. The size of the harmonics admittance matrix for this system will be of $4 \times 4$ (4), con-

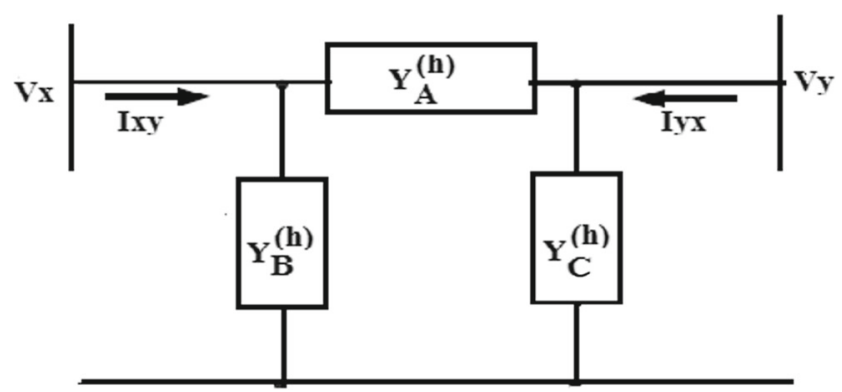

Fig. 4 Transformer $\pi$ equivalent harmonic model 
Fig. 5 Representation of three phase four wire distribution line

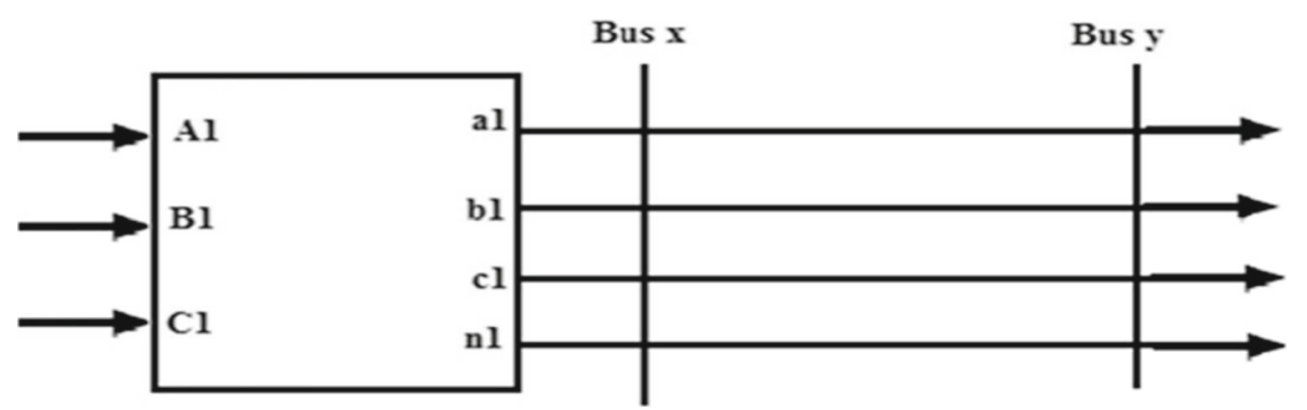

sisting of self and transfer admittances between phases and neutral $[26,27,29]$.

$\left[Y_{x y}^{(h)}\right]=\left[\begin{array}{cccc}y_{a a}^{(h)} & y_{a b}^{(h)} & y_{a c}^{(h)} & y_{a n}^{(h)} \\ y_{b a}^{(h)} & y_{b b}^{(h)} & y_{b c}^{(h)} & y_{b n}^{(h)} \\ y_{c a}^{(h)} & y_{c b}^{(h)} & y_{c c}^{(h)} & y_{c n}^{(h)} \\ y_{n a}^{(h)} & y_{n b}^{(h)} & y_{n c}^{(h)} & y_{n n}^{(h)}\end{array}\right]$

\section{Modeling of Load}

The harmonic power flow analysis requires equivalent circuits of linear loads because they affect the resonance conditions and provide damping at higher frequencies [30]. At fundamental frequency, linear loads are modeled as conventional PQ and PV buses. However, shunt admittances are used to model them at harmonic frequencies [28]. The admittance of a linear load connected to bus $y$ at the $h^{\text {th }}$ harmonic frequency is given in Eq. 5.

$y_{y}^{(h)}=\left(\frac{P_{y}^{(1)}-j \frac{Q_{y}^{(1)}}{h}}{\left|V_{y}^{(1)}\right|^{2}}\right)$
At the harmonic frequencies, the nonlinear loads (such as line commutated converters, arc furnace etc.) can be modeled as harmonic current sources and shunt admittance. The harmonic current at any bus $y$ will be function of its fundamental and harmonic voltages as expressed in Eq. 6.

$I^{(h)}=f_{y}^{h}\left(V_{y}^{(1)}, V_{y}^{(5)}, V_{y}^{(7)}, V_{y}^{(9)}, \ldots . . V_{y}^{(L)}, \alpha_{y}, \beta_{y}\right)$

Where, $L$ is the maximum harmonic order, $\alpha_{k}$ and $\beta_{k}$ are nonlinear load control parameters.

\section{Harmonic Analysis Algorithm}

The harmonic power flow is a reformulation of the conventional Newton-Raphson power flow algorithm to include non-linear loads [31]. In this section, the conventional Newton-Raphson algorithm is reformulated by including the solar PV and nonlinear loads. The solar PV can be modeled as a constant PQ model and negative load with current injecting into the node. For steady-state analysis, constant negative PQ model of DG resources can be used [32]. This method checks the power mismatch at the end of feeders and

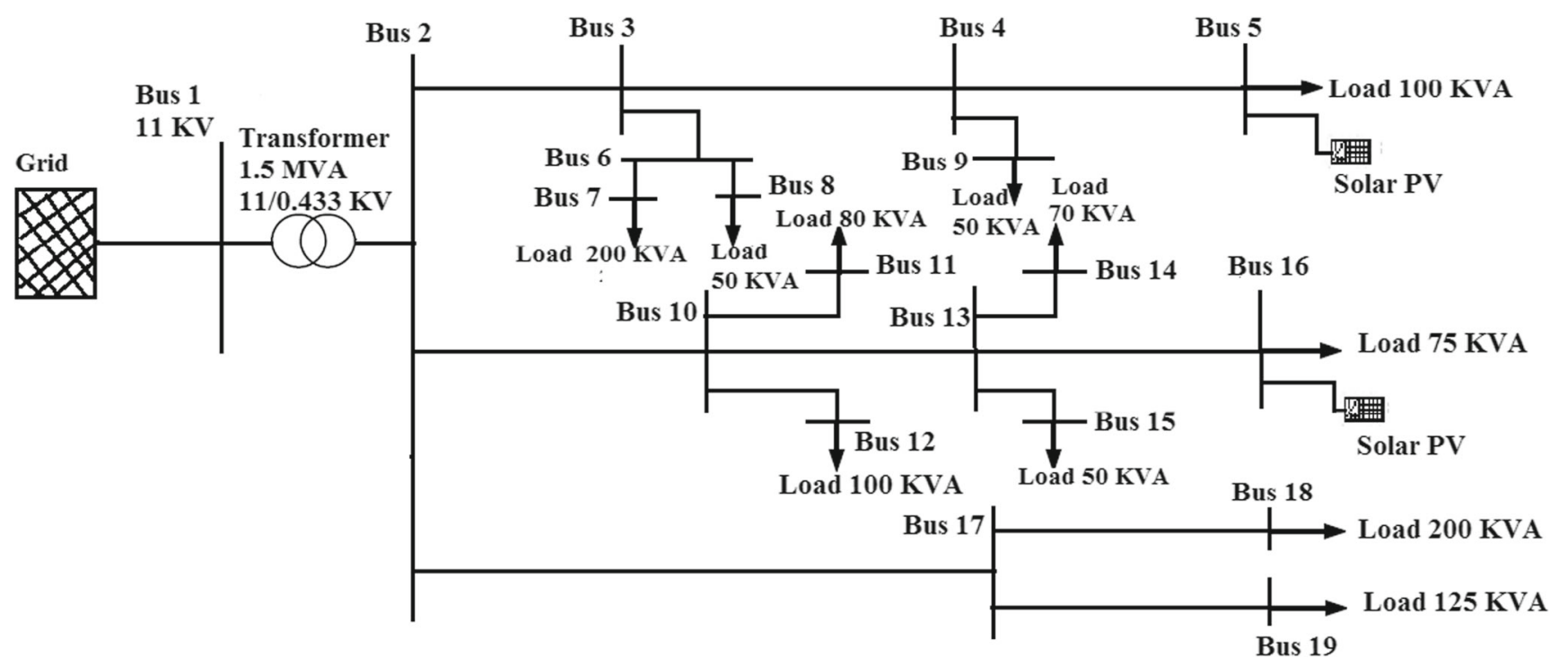

Fig. 6 Distribution grid with solar PV integration 
laterals, in order to find the harmonics and system operating variables of PV integrated distribution system [33, 34]. Initially, the variables are arranged into a real vector $Z$, which consists of fundamental, harmonic voltages and nonlinear solar PV elements.

$[Z]=\left[V^{(1)}, V^{(5)}, V^{(7)} \ldots \ldots V^{(L)},[\Omega]\right]^{T}$

In Eq. $7,[\Omega]$ are the nonlinear parameters of solar PV and $L$ is the maximum harmonic order. The algorithm steps for power flow solution are given below:

Step 1: Read the distribution networks line data and bus data. Implement the fundamental power flow analysis and compute an initial value for the fundamental bus voltage magnitudes and phase angles.

Step 2: Calculate the fundamental and harmonic current mismatch vector for solar PV and various load buses in Fig. 6.

$$
\begin{aligned}
& \Delta I^{(1)}=\left[\begin{array}{l}
I_{r, 1}^{(1)}, I_{i, 1}^{(1)}, \ldots \ldots \ldots I_{r, x-1}^{(1)}, I_{i, x-1}^{(1)}, \\
I_{r, x}^{(1)}+g_{r, x}^{(1)}, I_{i, x}^{(1)}+g_{i, x}^{(1)}, \ldots \\
I_{r, y}^{(1)}+g_{r, y}^{(1)}, I_{i, y}^{(1)}+g_{i, y}^{(1)}
\end{array}\right]^{T} \\
& \Delta I^{(h)}=\left[\begin{array}{l}
I_{r, 1}^{(h)}, I_{i, 1}^{(h)}, \ldots \ldots \ldots I_{r, x-1}^{(h)}, I_{i, x-1}^{(h)}, \\
I_{r, x}^{(h)}+g_{r, x}^{(h)}, I_{i, x}^{(h)}+g_{i, x}^{(h)}, \\
I_{r, y}^{(h)}+g_{r, y}^{(h)}, I_{i, y}^{(h)}+g_{i, y}^{(h)}
\end{array}\right]^{T}
\end{aligned}
$$

In Eq. $8, I_{r, x}^{(1)}$ and $I_{i, x}^{(1)}$ are the real and imaginary bus injection currents at bus $x$. Superscript (1) is used for fundamental frequency. $g_{r, x}^{(1)}$ and $g_{i, x}^{(1)}$ are real and imaginary line currents. Subscript $(y)$ is used for corresponding bus injection current at $y$ bus. In Eq. $9, I_{r, x}^{(h)}$ and $I_{i, x}^{(h)}$ are the real and imaginary bus injection currents at bus $x$. Superscript $(h)$ is used for harmonic frequency. $g_{r, x}^{(h)}$ and $g_{i, x}^{(h)}$ are real and imaginary line currents.

Step 3: Evaluate the mismatch vector for mismatch in power and current on each bus. Check the convergence.

$\Delta M=\left[\Delta W, \Delta I^{(5)}, \ldots \ldots . \Delta I^{(L)}, \Delta I^{(1)}\right]^{T}$

In Eq. 10, $\Delta W$ is the mismatch power vector and $\left(\Delta I^{(5)}, \ldots \ldots . \Delta I^{(L)}, \Delta I^{(1)}\right)$ is mismatch current vector for harmonics including fundamental.

Step 4: Evaluate jacobian matrix and calculate correction vector.

$$
J^{(h)}=\left[\begin{array}{cccc}
\frac{\partial \Delta P_{2}^{(h)}}{\partial \delta_{2}^{(h)}} & \frac{\partial \Delta P_{2}^{(h)}}{\partial V_{2}^{(h)}} \ldots \ldots . . \frac{\partial \Delta P_{2}^{(h)}}{\partial \delta_{y}^{(h)}} & \frac{\partial \Delta P_{2}^{(h)}}{\partial V_{y}^{(h)}} \\
\frac{\partial \Delta Q_{2}^{(h)}}{\partial \delta_{2}^{(h)}} & \frac{\partial \Delta Q_{2}^{(h)}}{\partial V_{2}^{(h)}} \ldots . . . & \frac{\partial \Delta Q_{2}^{(h)}}{\partial \delta_{y}^{(h)}} & \frac{\partial \Delta Q_{2}^{(h)}}{\partial V_{y}^{(h)}} \\
. . & . . & . . & . . \\
. . & . . & . . & . .
\end{array}\right]
$$

In Eq. $11, J^{(h)}$ is the jacobian matrix at harmonic frequency which has all entries zero for linear buses. $\Delta P$
Table 1 Solar PV parameters

\begin{tabular}{lll}
\hline 1 & PV panel (Watt/panel) & 280 \\
2 & PV panels in series & 11 \\
3 & PV panels in parallel & 161 \\
4 & Total No. of panels & 1771 \\
5 & Volts , dc & 400 \\
6 & KW, dc & 500 \\
7 & Amp, dc & 1240 \\
8 & Inverter, kva & 600 \\
9 & Inverter, amp & 800 \\
10 & Inverter, output voltage (volts) & 433 \\
\hline
\end{tabular}

and $\Delta Q$ are the change in real and reactive powers, w.r.t voltage $V$ and phase angle $\delta$.

Step 5: Compute the bus voltage correction vector as given in Eq. 12. $\eta$ is the number of iterations.

$$
\Delta Z=Z^{(\eta)}-Z^{(\eta+1)}
$$

Step 6: Update the vector $Z$ as given in Eq. 13 .

$$
Z^{(\eta+1)}=Z^{(\eta)}-\Delta Z
$$

Step 7: Update the real and reactive power mismatch due to solar PV and nonlinear loads at given buses.

$$
\begin{aligned}
& \Delta P_{k}^{\text {nonlinear }}=P_{k}^{(t)}+\sum_{h} F_{r, k}^{(h)} \\
& \Delta Q_{k}^{\text {nonlinear }}=Q_{k}^{(t)}+\sum_{h} F_{i, k}^{(h)}
\end{aligned}
$$

Table 2 Current THD in various branches

\begin{tabular}{llll}
\hline From & To & \% THD With PV & \% THD Without PV \\
\hline Bus1 & Bus2 & 45.07 & 3.91 \\
Bus2 & Bus3 & 50.18 & 1.05 \\
Bus2 & Bus 10 & 49.03 & 1.04 \\
Bus2 & Bus 17 & 15.57 & 2.44 \\
Bus3 & Bus4 & 39.22 & 1.07 \\
Bus3 & Bus6 & 8.55 & 1.03 \\
Bus4 & Bus5 & 35.92 & 1.09 \\
Bus4 & Bus 9 & 9.19 & 1.03 \\
Bus6 & Bus 7 & 8.55 & 1.03 \\
Bus6 & Bus 8 & 8.56 & 1.03 \\
Bus 10 & Bus 11 & 8.99 & 1.03 \\
Bus 10 & Bus 12 & 8.99 & 1.03 \\
Bus 10 & Bus13 & 40.84 & 1.06 \\
Bus 13 & Bus14 & 9.64 & 1.04 \\
Bus13 & Bus 15 & 9.69 & 1.04 \\
Bus13 & Bus 16 & 33.3 & 1.09 \\
Bus 17 & Bus 18 & 24.66 & 3.4 \\
Bus 17 & Bus 19 & 8.05 & 1.72 \\
\hline
\end{tabular}




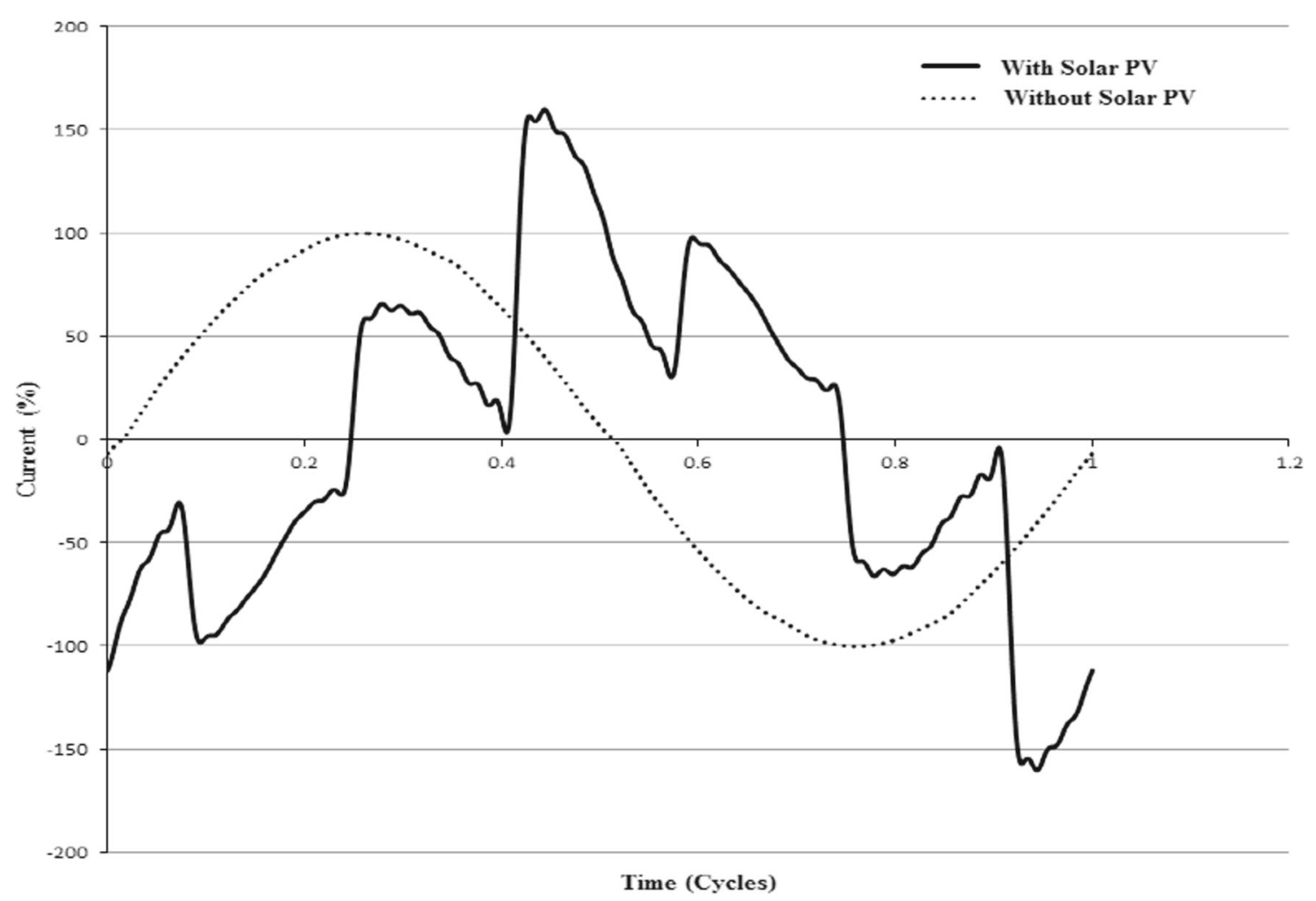

Fig. 7 Harmonic current waveform in the branch connected between buses 2 and 3

Fig. 8 Spectrum of harmonic current in the branch connected between buses 2 and 3

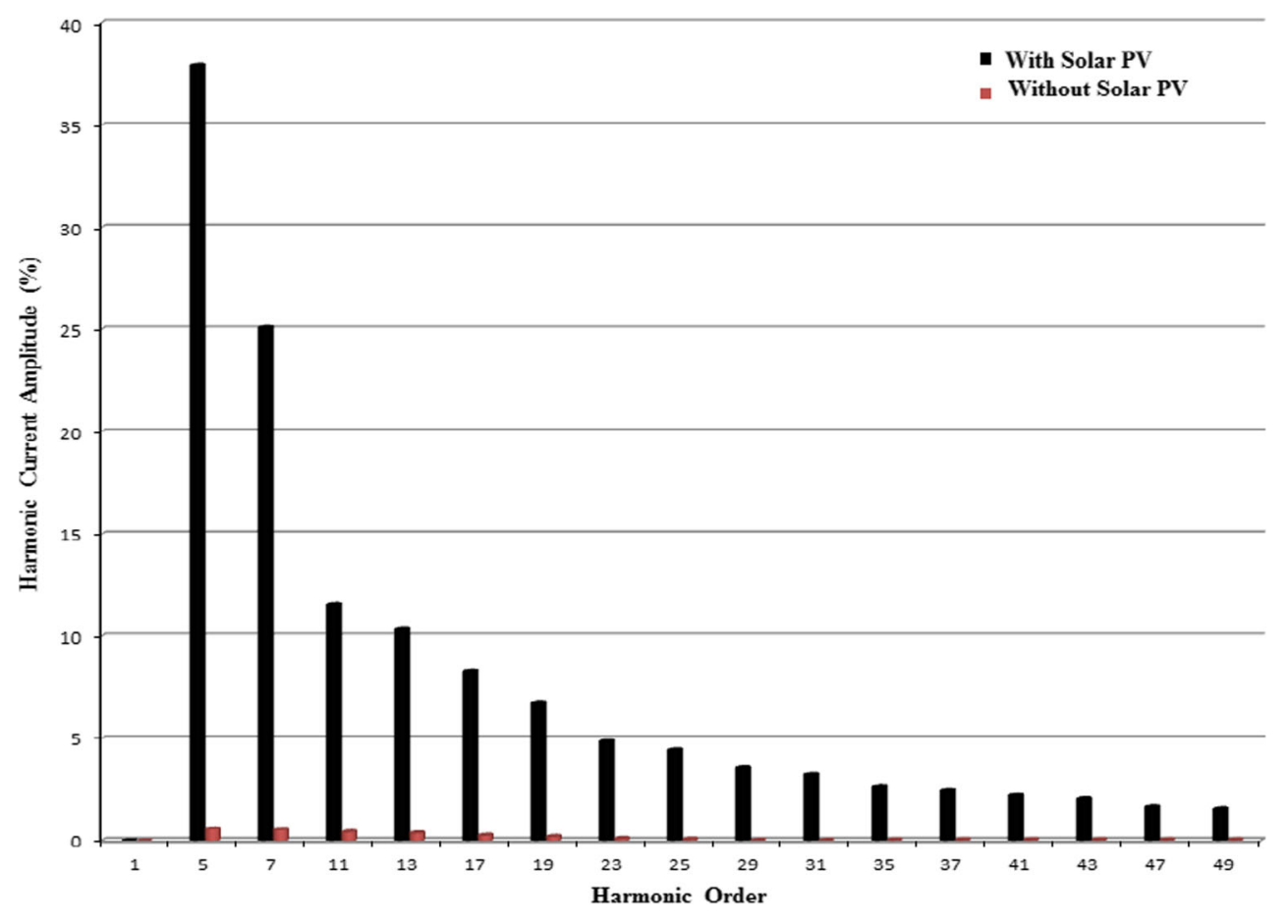


Fig. 9 Transformer harmonic current waveform

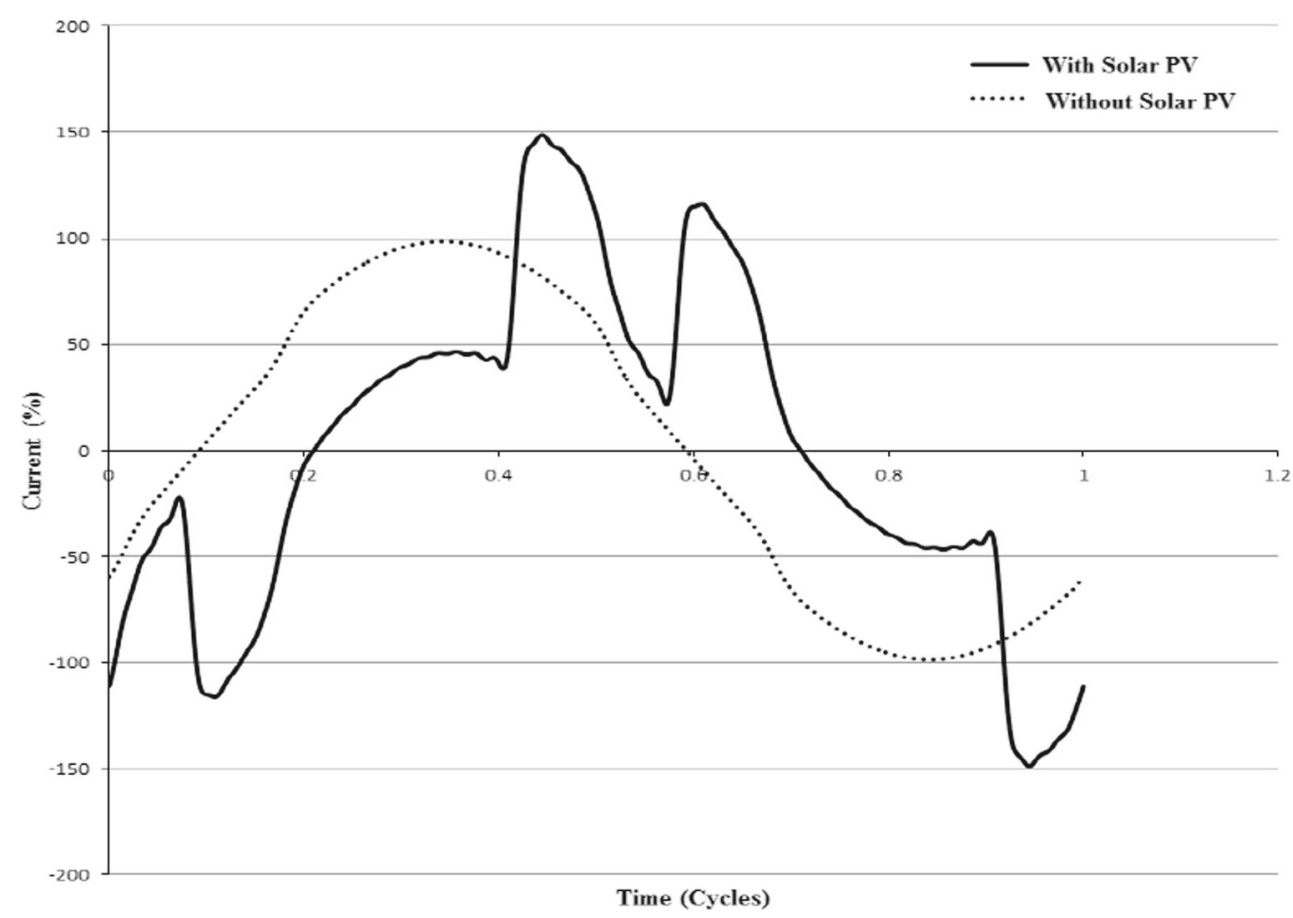

Where, $k=1,2,3,4, \ldots \ldots . x, y$.

In Eqs. $14-15, P_{k}$ and $Q_{k}$ are the total real and reactive power at bus $k . F_{r, k}$ and $F_{i, k}$ are the total real and imaginary line powers.

Step 8: Go to step 2. Calculate the mismatch vectors again and check the convergence.

\section{Design of Smart Distribution Grid System}

The solar PV integrated smart distribution grid system is modeled in Fig. 6. Loads are fed from an $11 \mathrm{KV}$ grid through a transformer of rating 1.5 MVA, 11/0.433 KV. Laterals are feeding the loads of varying nature in the area. Table 1
Fig. 10 Spectrum of transformer harmonic current

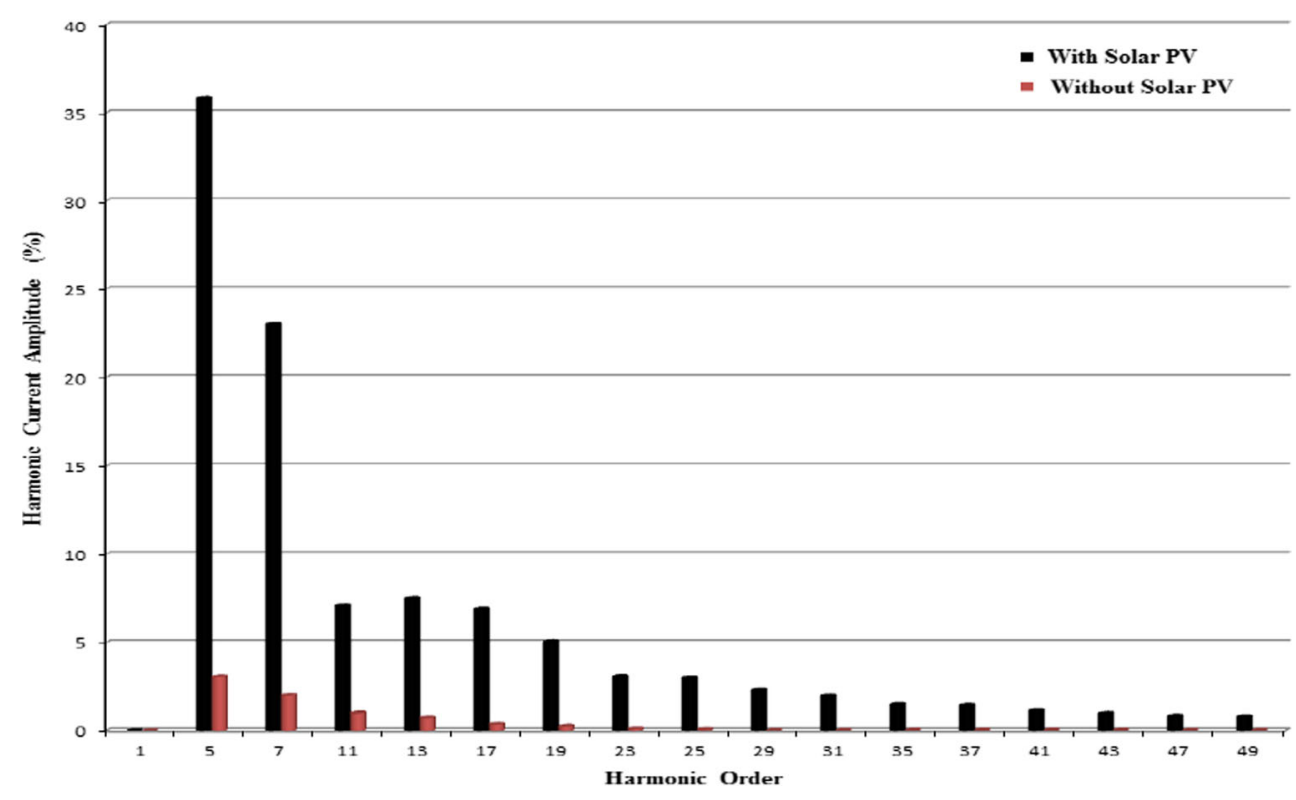


Table 3 Voltage THD at various buses

\begin{tabular}{lll}
\hline Bus No. & \% THD With PV & \% THD Without PV \\
\hline 1 & 0.57 & 0.08 \\
2 & 9.81 & 1.28 \\
3 & 10.58 & 1.28 \\
4 & 11.28 & 1.28 \\
5 & 13.19 & 1.28 \\
6 & 10.48 & 1.28 \\
7 & 10.48 & 1.28 \\
8 & 10.48 & 1.28 \\
9 & 11.26 & 1.28 \\
10 & 11.08 & 1.28 \\
11 & 11.05 & 1.28 \\
12 & 11.04 & 1.28 \\
13 & 11.76 & 1.28 \\
14 & 11.74 & 1.28 \\
15 & 11.75 & 1.28 \\
16 & 13.67 & 1.28 \\
17 & 10.03 & 2.15 \\
18 & 10.25 & 2.61 \\
19 & 10 & 2.14 \\
\hline & &
\end{tabular}

presents the parameters of solar PV which are connected at bus 5 and 16 respectively.

\section{Simulation Results Analysis}

This section presents the extensive analysis of harmonics with and without solar PV integration at the various buses and branches of the modeled smart distribution grid system. The branch wise comparison of total harmonic distortions in current, with and without solar PV integration, has been presented in Table 2.

It is found that the harmonic distortion in current rises sharply after solar PV integration. The current harmonic distortion (\%THD) in the branch connected between buses 2-3 increases to a very high value of $50.18 \%$. The nature of such distorted current is shown in Fig. 7. Analysis of harmonic amplitude spectrum, as shown in Fig. 8 depicts that it contains the $5^{\text {th }}, 7^{\text {th }}, 11^{\text {th }}, 13^{\text {th }}, 17^{\text {th }}$, and $19^{\text {th }}$ order harmonics, which violates the acceptable limits as given in Table 6 . The share of $5^{\text {th }}$ and $7^{\text {th }}$ order harmonic is more, which affects the system performance severely and needs immediate filtering.

Harmonics makes the distribution transformer current highly distorted as shown in Fig. 9. Amplitude spectrum in Fig. 10 contains the $5^{\text {th }}, 7^{\text {th }}, 11^{\text {th }}, 13^{\text {th }}, 17^{\text {th }}$ and $19^{\text {th }}$ order harmonics which violates the standard limits given in Table 6. Such current escalate the iron losses and reduces the transformer efficiency, consequently derating becomes essential.
Fig. 11 Voltage waveform at bus 16

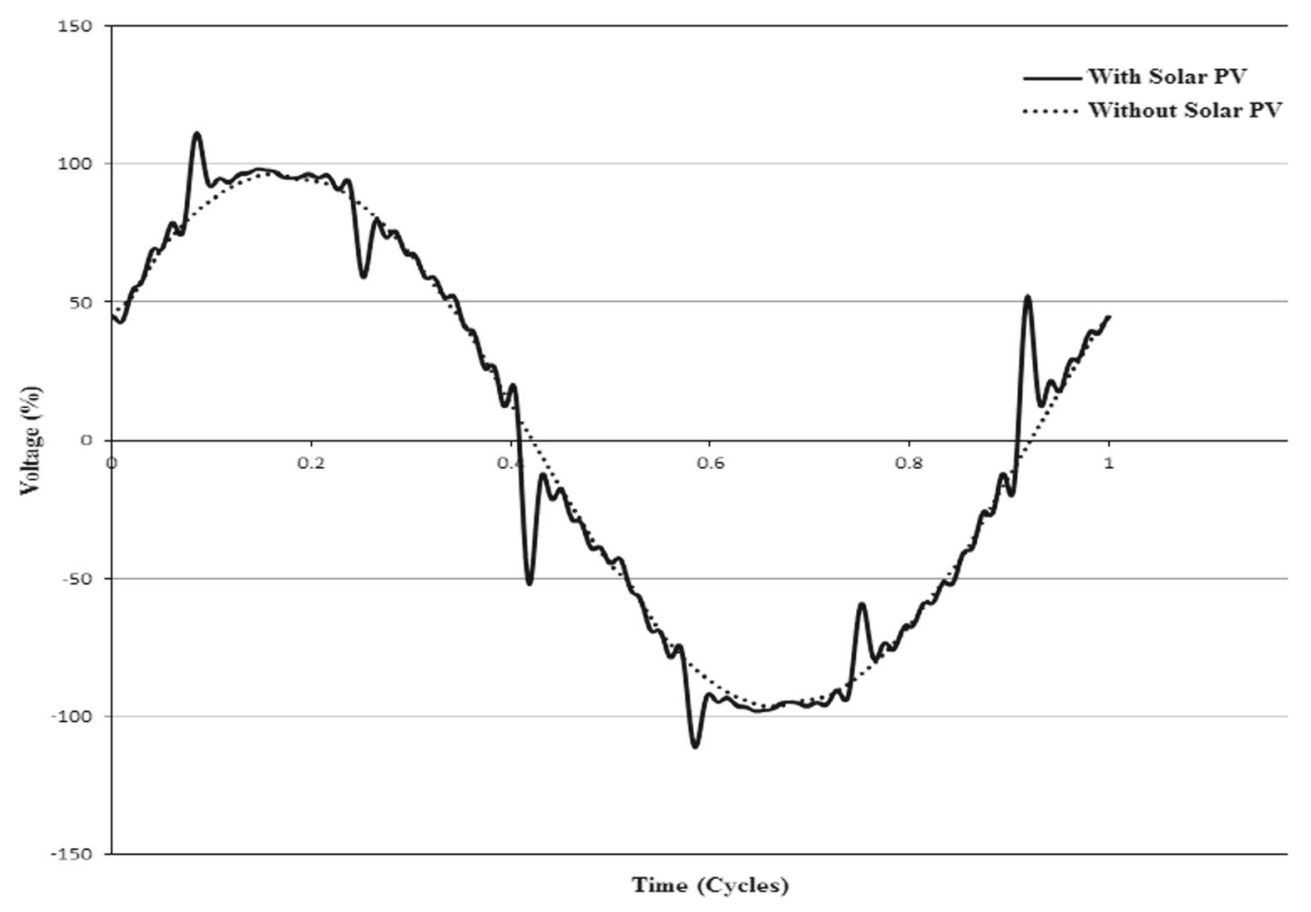


Fig. 12 Spectrum of harmonic voltage at bus 16

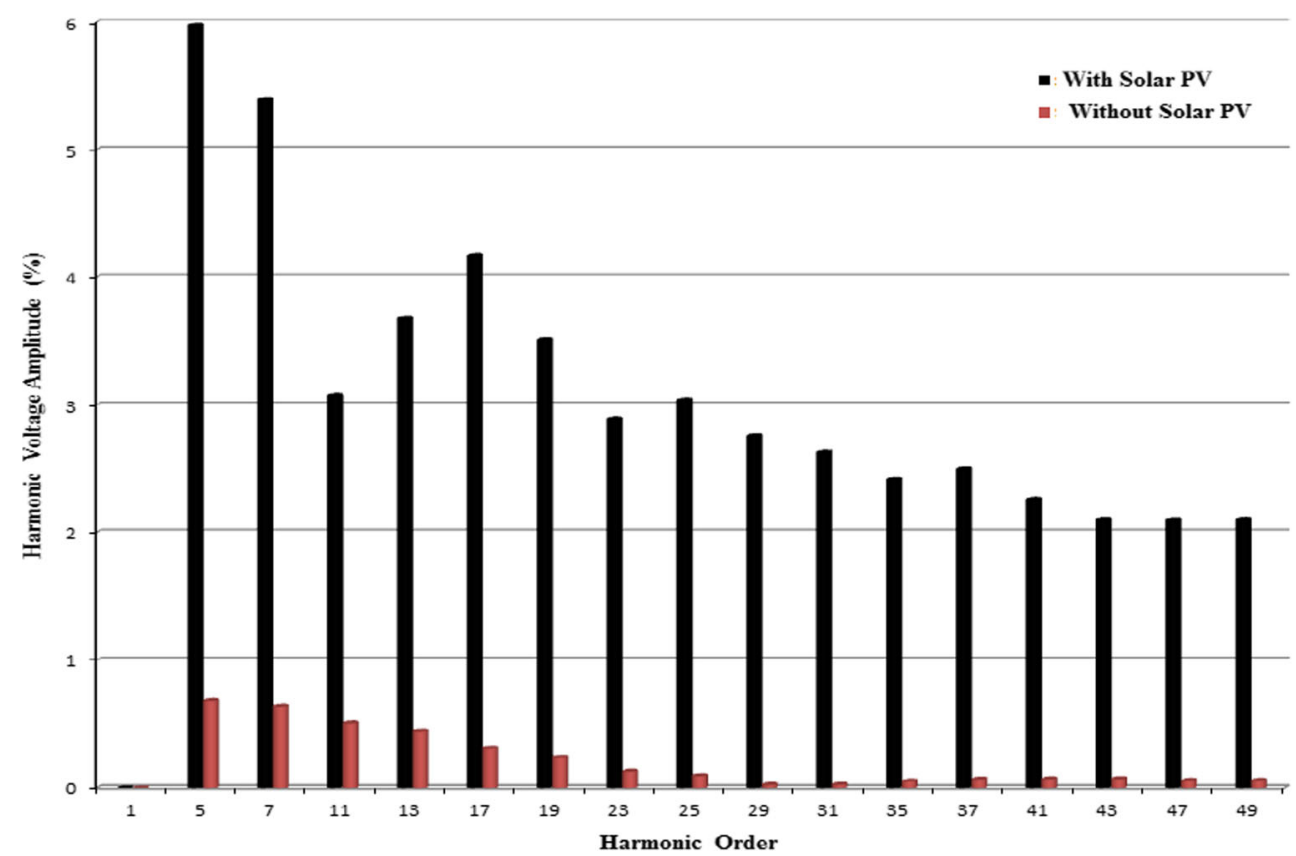

The bus wise comparison of total harmonic distortions in voltage, with and without solar PV integration, has been

Table 4 Variation in voltage due to harmonics after solar PV integration

\begin{tabular}{lllll}
\hline Bus ID & Specified kV & $\begin{array}{l}\text { Fundamental } \\
\text { Voltage } \%\end{array}$ & RMS Voltage \% & THD \% \\
\hline Bus 1 & 11 & 100 & 100.0 & 0.57 \\
Bus 2 & 0.433 & 97.7 & 98.2 & 9.81 \\
Bus 3 & 0.433 & 97.6 & 98.1 & 10.58 \\
Bus 4 & 0.433 & 97.7 & 98.3 & 11.28 \\
Bus 5 & 0.433 & 98.1 & 98.9 & 13.19 \\
Bus 6 & 0.433 & 96.7 & 97.2 & 10.48 \\
Bus 7 & 0.433 & 96.5 & 97.0 & 10.48 \\
Bus 8 & 0.433 & 96.5 & 97.1 & 10.48 \\
Bus 9 & 0.433 & 96.5 & 97.1 & 11.26 \\
Bus 10 & 0.433 & 97.6 & 98.21 & 11.08 \\
Bus 11 & 0.433 & 96.2 & 96.8 & 11.05 \\
Bus 12 & 0.433 & 96.2 & 96.8 & 1.04 \\
Bus 13 & 0.433 & 97.6 & 98.3 & 11.76 \\
Bus 14 & 0.433 & 97.0 & 97.6 & 11.74 \\
Bus 15 & 0.433 & 97.3 & 97.9 & 11.75 \\
Bus 16 & 0.433 & 98.1 & 99.0 & 13.67 \\
Bus 17 & 0.433 & 96.7 & 97.2 & 10.03 \\
Bus 18 & 0.433 & 96.4 & 96.9 & 10.25 \\
Bus 19 & 0.433 & 95.4 & 95.9 & 10 \\
\hline
\end{tabular}

presented in Table 3. Results show that with solar PV integration, the THD at bus 16 rises to a very high value equal

Table 5 System power flow variations after solar PV integration

\begin{tabular}{llll}
\hline Bus ID & $\begin{array}{l}\text { Variation in } \\
\text { MW }(\%)\end{array}$ & $\begin{array}{l}\text { Variation in } \\
\text { MVAR }(\%)\end{array}$ & Variation in Amp (\%) \\
\hline Bus 1 & 97 & -2 & 46 \\
Bus 2 & 133 & -3 & 39 \\
Bus 3 & 363 & -5 & -128 \\
Bus 4 & 0 & -4 & 0 \\
Bus 5 & 556 & -8 & -285 \\
Bus 6 & -1 & 0 & 0 \\
Bus 7 & -1 & -1 & 0 \\
Bus 8 & 0 & -4 & 0 \\
Bus 9 & 0 & 0 & 0 \\
Bus 10 & -2 & 0 & 0 \\
Bus 11 & 0 & 0 & 0 \\
Bus 12 & -1 & -2 & 0 \\
Bus 13 & 275 & -5 & -58 \\
Bus 14 & -2 & 0 & 0 \\
Bus 15 & -2 & 0 & 0 \\
Bus 16 & 744 & -11 & -442 \\
Bus 17 & -1 & -1 & -1 \\
Bus 18 & -1 & -1 & -1 \\
Bus 19 & 0 & 0 & 0 \\
\hline & & & \\
\hline
\end{tabular}


Table 6 Limits of maximum harmonic current distortion inPercent, (Based on IEEE Std. 519-1992, 1993) [11]

\begin{tabular}{ll}
\hline Harmonic Orders & IEEE Std. 519-1992(\%) \\
\hline 5 & 4 \\
7 & 4 \\
11 & 2 \\
13 & 2 \\
17 & 1.5 \\
19 & 1.5 \\
23 & 0.6 \\
25 & 0.6 \\
29 & 0.6 \\
31 & 0.6 \\
35 & 0.3 \\
THD & 5 \\
\hline
\end{tabular}

to $13.67 \%$ as compared to the THD value of $1.28 \%$ without solar PV, Moreover, all other buses are also affected by harmonics. A clear violation of $5 \%$ voltage standard limits, as given in Table 7 can be seen after solar PV integration.

The distorted voltage waveform at bus 16 is presented in Fig. 11. The individual voltage harmonic contribution is depicted in concerned harmonic spectrum Fig. 12. It is found that, the harmonic spectrum contains $5^{\text {th }}, 7^{\text {th }}, 13^{\text {th }}$, $17^{\text {th }}$, and $19^{\text {th }}$ order harmonics which violates the individual harmonic distortion limits of $3 \%$. Moreover, these harmonic distortions alter the value of fundamental and RMS voltages as presented in Table 4 . Therefore, a proper mitigation technique is essential to filter out these harmonics and resolve the related issues.

Table 5 presents the comparative results of harmonic power flow variables. The variation in current is very high at buses 5 and 16 after solar PV integration, which poses burden on the connecting cables and requires higher size cables. Moreover, negative variation in power flow and current is observed at some buses. Such reverse power can affect the protection system and false tripping of protective gears can occur (Tables 6 and 7).

Table 7 Limits of harmonic voltage distortion at the PCC (Based on IEEE Std. 519-1992, 1993) [11]

\begin{tabular}{lll}
\hline PCC Voltage & $\begin{array}{l}\text { Individual Harmonic } \\
\text { Magnitude (\%) }\end{array}$ & $\begin{array}{l}\text { Total Harmonic } \\
\text { Distortion (THD) }\end{array}$ \\
\hline$\leq 69 \mathrm{KV}$ & 3.0 & 5.0
\end{tabular}

\section{Conclusion}

The power distribution system has a large number of nonlinear loads, which generates harmonics in the system. Integration of solar PV further enhances the distortion and thereby degrades the quality of power supply. Mitigation of harmonics is possible only if their exact nature, individual percentage and THD are known. Normal power flow method does not provide above information. In this paper, harmonic power flow approach is implemented for a solar PV integrated smart distribution system. Comparison results of before and after solar PV integration has been analyzed. It is observed that integration of solar PV alters the system power flow variables and increases the harmonics in the system beyond the prescribed limit, which is a major challenge and needs to be addressed in the integration process. The grid must be able to absorb these challenges in order to achieve the smart grid objectives. The analysis presented in this paper will be helpful for designing an electronic filter as a control solution and make the system harmonic free.

\section{References}

1. Louie KW, Wilson P, Rivas RA, Wang A, Buchanan P (2006) Discussion on power system harmonic analysis in the frequency domain. In: Proceedings of the IEEE conference PES transmission and distribution and exposition Latin America, pp 1-6

2. Sidrach-De-Cardona M, Carretero J (2005) Analysis of the current total harmonic distortion for different single-phase inverters for grid-connected pv-systems. Sol Energy Mater Sol Cells 87:529540

3. Schlabbach J, Kammer L (2006) Prediction of harmonic currents of PV inverters using measured solar radiation data. In: Proceedings of the IEEE conference mediterranean electrotechnical Spain, pp 857-860

4. Han Bao, Shaoqing Ying, Harald Schwarz (2014) Harmonic impact of decentralized photovoltaic systems and limitation of photovoltaic capacity in low voltage grid. In: Proceedings of the IEEE conference environment and electrical engineering, pp $1-4$

5. Heskes PJM, Enslin JHR (2003) Power quality behavior of different photovoltaic inverter topologies. In: Proceedings of the 24 th power conversion \&, intelligent motion conference Nurnberg, pp $1-10$

6. Benhabib MC, Myrzik JMA, Duarte JL (2007) Harmonic effects caused by large scale PV installations in LV network. In: Proceedings of the 9th international conference electrical power quality and utilization Barcelona, Spain, pp 1-6

7. Papaioannou IT et al. (2008) Harmonic impact of small photovoltaic systems connected to the LV distribution network. In: Proceedings of the 5th international conference european electricity market Lisbon, Portugal, pp 1-6

8. Macedo WN, Zilles R (2009) Influence of the power contribution of a grid-connected photovoltaic system and its operational particularities. Energy Sustain Dev 13(3):202-211

9. Haitao $\mathrm{H}$ et al. (2015) Potential harmonic resonance impacts of PV inverter filters on distribution systems. Proc IEEE Trans Sustainable Energy 6(1):151-161 
10. Infield DG et al. (2004) Power quality from multiple grid connected single-phase inverters. Proc IEEE Trans Power Delivery 19(4):1983-1989

11. IEEE Std 519-1992 (1993) IEEE recommended practice and requirement for harmonic control in electrical power systems. IEEE. ISBN 1-55937-239-7

12. Nelson J (2003) The physics of solar cells. Text book published by Imperial College Press, London

13. Mazer JA (1997) Solar cells: an introduction to crystalline photovoltaic technology. Text book published by Kluwer Academic Publishers, Boston

14. Wilfried GJHM, Van S, Lars K, Francesco R (2012) Physics and technology of amorphous-crystalline hetero structure silicon solar cell. Text book published by Springer, Berlin

15. Villalva MG, Gazol JR, Filho ER (2009) Comprehensive approach to modeling and simulation of photovoltaic arrays. Proc IEEE Trans Power Electronics 24(5):1198-1208

16. Kumar RA, Suresh MS, Nagaraju J (2006) Effect of solar array capacitance on the performance of switching shunt voltage regulator. Proc IEEE Trans Power Electronics 21(2):543-548

17. Benavides ND, Chapman PL (2008) Modeling the effect of voltage ripple on the power output of photovoltaic modules. Proc IEEE Trans Industrial Electronics 55(7):2638-2643

18. Xu W (2001) Component modeling issues for power quality assessment. Proc IEEE Power Engineering Review:12-17

19. IEEE Task force on Harmonics Modeling and Simulation (1996) The modeling and simulation of the propagation of harmonics in electric power networks Part I: Concepts, models and simulation techniques. Proc IEEE Trans Power Delivery 11(1):452-465

20. Neves WLA, Dommel HW, Xu W (1995) Practical distribution transformer models for harmonic studies. Proc IEEE Trans Power Delivery 10(2):906-912

21. Das JC (2002) Power system analysis: short-circuit load flow and harmonics. Text book published by CRC, Boca Raton

22. Greene JD, Gross CA (1988) Nonlinear modeling of transformers. Proc IEEE Trans Industry Applications 24(3):434-438

23. IEEE Task Force on harmonics modeling and simulation (1998) Tutorial on harmonics modeling and simulation. IEEE Power Engineering Society
24. Arrillaga J et al. (1997) Power system harmonics. Text book published by John Wiley \& Sons, New York

25. Kusic G (2008) Computer-aided power systems analysis. Text book published by CRC Press Taylor \& Francis Group, New York

26. Wortman MA, Allen DL, Grigsby LL (1985) Techniques for the steady state representation of unbalanced power systems: Part I. A systematic building block approach to network modeling. Proc IEEE Trans Power Apparatus Systems 104(10): 2805-2814

27. Halpin SM, Mai A, Dickinson CR (1996) Calculation of harmonic current flows in single-phase power distribution systems. In: Proceedings of the 7th ieee international conference on harmonics and the quality of power, pp 516-521

28. Lu N et al. (2008) Load component database of household appliances and small office equipment. In: Proc IEEE Power and Energy Society General Meeting conversion and delivery of electrical energy in the 21st century, pp 1-5

29. Alam MJE, Muttaqi KM, Sutanto D (2013) A three-phase power flow approach for integrated 3-Wire MV and 4-Wire multi grounded LV networks with rooftop solar PV. Proc IEEE Trans Power Systems 28(2):1728-1737

30. Capasso A, Prudenzi A, Lamedica R (1989) Equivalents of complex loads for harmonic penetration studies. In: Proceedings of the international symposium Electric Energy Conversion in Power Systems Capri Conference Italy

31. Xia D, Heydt GT (1982) Harmonic power flow studies, part I- formulation and solution. Proc IEEE Trans Power Apparatus System 101(6):1257-1265

32. Papanikolau NP, Tatakis EC, Kyritsis AC (2009) Analytical model for PV distributed generators suitable for power systems studies. In: Proceedings of the 13th European Conference on Power Electronics and Applications, pp 1-10

33. Fuchs E, Masoum M (2008) Power quality in power systems and electrical machines. Text book published by Academic Press Elsevier

34. Arrillaga J, Watson Neville R, Bathurst Graeme N (2004) A multi frequency power flow of general applicability. Proc IEEE Trans Power Delivery 19(1):342-349 